\title{
Gestação em pacientes com síndrome de Klippel-Trenaunay: revisão da literatura
}

\section{Pregnancy in Klippel-Trenaunay syndrome: review of literature}

\author{
Aleylove Talans', Alan Saito Ramalho', Maria Rita de Figueiredo Lemos Bortolotto², \\ Rossana Pulcineli Vieira Francisco ${ }^{3}$, Marcelo Zugaib ${ }^{4}$
}

Talans A, Ramalho AS, Bortolotto MRFL, Francisco RPV, Zugaib M. Gestação em pacientes com síndrome de Klippel-Trenaunay: revisão da literatura. Rev Medicina (São Paulo). 2010 abr.-jun.;89(2):93-100.

\begin{abstract}
RESUMO: A síndrome de Klippel-Trenaunay é uma anomalia congênita de etiologia desconhecida, caracterizada pela presença de malformações venosas e arteriais, hipertrofia de ossos e tecidos moles. São raros os relatos de gravidez em pacientes portadoras dessa síndrome, sendo associada com alto risco obstétrico. Objetivo: Resumir os conhecimentos acumulados sobre gestação em pacientes com síndrome de Klippel-Trenaunay, bem como sobre a própria síndrome, para permitir que essas pacientes sejam prontamente reconhecidas e recebam os cuidados necessários para um bom desfecho obstétrico. Método: Foi realizada pesquisa na literatura em língua inglesa, até outubro de 2009, na base de dados Medline e Cochrane Library. Resultados: Foram encontrados 17 casos relatados na literatura de gestações em pacientes com síndrome de Klippel-Trenaunay. A evolução obstétrica das gestantes é variável e envolve eventos adversos, com piora das manifestações prévias, hemorragia, coagulação intravascular disseminada, eventos tromboembólicos e dor. Conclusão: A gestação agrava o risco de eventos adversos. O entendimento da síndrome e cuidado multidisciplinar pré-natal, intra e pós-parto são fundamentais para o bom desfecho da gestação.
\end{abstract}

DESCRITORES: Síndrome de Klippel-Trenaunay-Weber/etiologia; Anomalia congênita; Gravidez de alto nível.

\section{INTRODUÇÃO}

A síndrome de Klippel-Trenaunay, também conhecida como síndrome de KlippelTrenaunay-Weber, é uma anormalidade congênita rara de manifestação clínica variável e de etiologia desconhecida. Embora tenha sido descrita em 1900 pelos médicos franceses Klippel e Trenaunay ${ }^{22}$, a falta de conhecimento e experiência sobre a etiologia e a patogênese da síndrome de Klippel-Trenaunay impede o consenso entre clínicos em relação ao manejo de pacientes com a síndrome ${ }^{4}$. O critério diagnóstico clássico é uma tríade de anormalidades, que incluem malformações capilares (comumente nevus cutâneos/

\footnotetext{
1. Acadêmico do 5ำ ano de Medicina da Faculdade de Medicina da Universidade de São Paulo.

2. Médica Supervisora do Departamento de Obstetrícia do Hospital das Clínicas da Faculdade de Medicina da Universidade de São Paulo.

3. Professora Doutora do Departamento de Obstetrícia da Faculdade de Medicina da Universidade de São Paulo.

4. Professor Titular do Departamento de Obstetrícia da Faculdade de Medicina da Universidade de São Paulo.

Endereço para correspondência: Aleylove Talans. Rua Caconde, 128 casa 6 - CEP: 01425-010. São Paulo, SP, e-mail: aleytalans@gmail.com
} 
Talans A, et al. Gestação em pacientes com síndrome de Klippel-Trenaunay.

hemangiomas), malformações venosas ou veias varicosas, e hipertrofia óssea e de tecidos moles afetando um ou mais membros, sem a presença de malformações arteriovenosas hemodinamicamente significativas ${ }^{3,8,22}$. O diagnóstico de síndrome de Klippel-Trenaunay é feito com a presença de, no mínimo, dois desses três sinais ${ }^{8,20,35}$. No entanto, a associação de anomalias venosas como hipoplasia, aplasia, incompetência venosa, e malformações linfáticas pode ocorrer com frequência ${ }^{20}$.

Gravidez é raramente descrita em pacientes com síndrome de Klippel-Trenaunay e sua incidência não é conhecida. Uma vez que a gravidez pode exacerbar as complicações da doença, colocando assim as gestantes sob alto risco obstétrico, a gestação é, historicamente, desencorajada nessas pacientes. Dessa forma, existe uma falta de conhecimento sobre a gestação em pacientes com síndrome de Klippel-Trenaunay.

Diante disso, foi realizada uma revisão do conhecimento atual tanto sobre a síndrome de Klippel-Trenaunay quanto sobre o manejo obstétrico de gestantes com essa condição.

\section{OBJETIVO}

O objetivo dessa revisão é resumir os conhecimentos acumulados sobre gestação em pacientes com síndrome de Klippel-Trenaunay, bem como sobre a própria síndrome, para permitir que essas pacientes sejam prontamente reconhecidas e recebam a atenção e os cuidados necessários para um bom desfecho obstétrico.

\section{MÉTODO}

Foi realizada extensa pesquisa na literatura em língua inglesa, até outubro de 2009, na base de dados Medline, utilizando-se os termos $\mathrm{MeSH}$ a seguir: "klippel-trenaunay", "klippel-trenaunay-weber", "klippel", "syndrome", "pregnancy", "pregnant", "obstetric" e "parturient". Foram realizados 32 cruzamentos entre esses termos, selecionando-se todos os artigos relevantes. A base de dados da Cochrane Library, até outubro de 2009, também foi pesquisada, utilizando-se os termos "klippeltrenaunay", "syndrome" e "pregnancy". Foram encontrados apenas 17 relatos de casos de gestações em pacientes com síndrome de Klippel-Trenaunay. As referências de cada artigo selecionado foram revisadas, extraindo-se outros artigos pertinentes. Artigos de arquivos pessoais dos autores também foram usados nesta revisão.

\section{REVISÃO DA LITERATURA}

\section{Etiologia}

A etiologia da síndrome de Klippel-Trenaunay permanece desconhecida ${ }^{2,8}$, embora existam algumas teorias de sua patogenia. Klippel e Trenaunay pensaram que as lesões estavam distribuídas em dermátomos e assim propuseram que uma anomalia do cordão espinhal seria a causa ${ }^{27,31}$. Várias outras hipóteses foram aventadas: malformações venosas congênitas que levam à hipertensão venosa crônica ${ }^{34}$, lesão intra-uterina aos gânglios simpáticos fetais ou ao trato nervoso lateral intermediário, causando perda crônica do tônus simpáticos e shunts arteriovenosos levando à hipertrofia e hemangiomas característicos da síndrome de Klippel-Trenaunay ${ }^{7}$, agressão vascular intra-útero durante a diferenciação vascular ${ }^{25}$. Recentemente, tem sido sugerido que a síndrome de Klippel-Trenaunay é causada por uma anormalidade mesodérmica que provoca comunicação arteriovenosa persistente a nível microscópico ${ }^{3,8,22}$.

\section{Epidemiologia}

A síndrome de Klippel-Trenaunay distribuise igualmente entre os diversos grupos étnicos e afeta mais homens, na proporção de 1,5: 1, 1,8,12,25,26. Apesar de centenas de casos de síndrome de KlippelTrenaunay terem sido descritos, somente houve descrição de 17 casos na literatura de gestação em mulheres com síndrome de Klippel-Trenaunay. Os casos relatados no presente estudo são $018^{\circ}$ e $19^{\circ}$ casos descritos na literatura.

\section{Genética}

O consenso geral é de que a maioria dos casos da síndrome de Klippel ocorre esporadicamente, sem um padrão definido de hereditariedade. Existem alguns relatos de recorrências familiares, mas o risco de transmissão da síndrome da mãe para seu filho é desconhecido ${ }^{26}$. Existem evidências de aumento na prevalência de hemangioma em familiares de pessoas com a síndrome de Klippel-Trenaunay ${ }^{1,26}$, e há várias teorias para tentar explicar o porquê disso. Tanto Aevolet et al. ${ }^{1}$ como Hergesell et al. ${ }^{19}$ acreditam que se trata de uma herança multifatorial, com penetração e expressão variáveis, enquanto LordaSanchez et al. ${ }^{24}$ e Ceballos-Quintal et al. ${ }^{9}$ sugerem que uma herança autossômica dominante é mais provável. Happle et al. ${ }^{17}$ acreditam numa explicação alternativa, de que os indivíduos herdam um dos genes e adquirem a outra mutação gênica. Houve 
um caso de síndrome de Klippel-Trenaunay causado pela translocação 5:11 relatado na literatura ${ }^{40}$.

\section{Sinais e sintomas}

O diagnóstico é clínico e pode ser feito por uma tríade de anormalidades, sendo necessários apenas dois dos seguintes para o diagnóstico da síndrome de Klippel-Trenaunay: malformações capilares; malformações venosas ou veias varicosas; e hipertrofia de ossos e tecidos moles, afetando um ou mais membros, sem comprometimento hemodinâmico significativo. Em $67 \%$ dos casos, os portadores da síndrome apresentam a tríade de sinais. Em 37\%, apenas dois deles. Geralmente, apenas uma extremidade é afetada, em sua maioria a perna ${ }^{36,41}$.

\section{Malformações capilares}

A malformação capilar mais comum é o nevo cutâneo, mas hemangiomas cutâneos e subcutâneos também são comuns. Os nevos manifestam-se em cerca de $2 / 3$ dos casos da síndrome ${ }^{15,36}$, são aparentes ao nascimento e tornamse mais proeminentes na puberdade e na gravidez, e geralmente afetam o membro hipertrofiado. O nevo, que resulta da estase de veias e capilares na derme, tem entre suas complicações o sangramento e a infecção, especialmente se a derme tiver veias ou vasos linfáticos ${ }^{11,20}$.

Os hemangiomas subcutâneos ocorrem em $21 \%$ dos casos. Podem acometer músculos, ossos, parede abdominal, coluna lombar e órgãos viscerais (como bexiga, baço, fígado, cólon, artérias renais e útero). Dentre as complicações, destacam-se: sangramento e hematúria ${ }^{8,26}$.

A gravidez cursa com o aumento da progesterona, do volume sanguíneo e do peso, levando frequentemente à piora das malformações capilares $^{34}$.

\section{Hipertrofia}

Cerca de $85 \%$ dos pacientes com a síndrome de Klippel-Trenaunay irão exibir hipertrofia de ossos e tecidos moles ${ }^{21}$. Ao nascimento, algum grau de hipertrofia já está presente, com progressão geralmente até os 12 anos $^{34,41}$. Se houver a presença de fístulas arteriovenosas, a disparidade entre 0 membro afetado e o contralateral é maior. Além das preocupações estéticas, a hipertrofia pode levar a dificuldade de marcha, escoliose e dor ${ }^{25}$.

\section{Malformações vasculares}

São geralmente varizes, presentes ao nascimento e que progridem com a deambulação ${ }^{11,20}$.
Uma grande varicosidade lateral, começando no pé, passando lateralmente ao joelho e então migrando medialmente ao nível da coxa, é muito comum entre portadores da síndrome, sendo frequentemente denominada de veia de Klippel-Trenaunay ${ }^{5,26}$.

Apesar das veias femoral e poplítea estarem frequentemente envolvidas, as varizes também ocorrem em veias perineais, suprapúbicas, cava inferior $^{19,26,41}$, e na veia ilíaca interna, levando a varicosidades ao redor do cólon, reto, útero e bexiga - por caminhos vasculares compensatórios.

A congestão dessas veias é agravada pelo aumento de níveis hormonais, de volume sanguíneo e de peso resultantes da gravidez. Essas malformações vasculares são associadas à tromboflebite, celulite, linfedema, úlcera de estase e dor ${ }^{20,26}$.

\section{Achados clínicos adicionais}

\section{Coagulopatia}

Pacientes portadores da síndrome de Klippel-Trenaunay têm um risco aumentado de trombose venosa profunda e outros fenômenos tromboembólicos. A frequência de TVP e eventos tromboembólicos pode chegar a até $17 \%$ em pacientes com a síndrome de Klippel-Trenaunay, muito superior à estimada incidência de 160 casos de trombose venosa profunda e de 60 casos de embolia pulmonar por ano para cada 100 mil habitantes nos países ocidentais ${ }^{5,15,28}$. Baskervile et al. ${ }^{5}$ relatou um índice de complicações tromboembólicas pós-operatórias em pacientes com a síndrome de Klippel-Trenaunay dez vezes maior que em outros pós-operatórios. Isso é provavelmente devido ao retorno venoso inadequado, condição agravada pela gravidez. Uma anormalidade no sistema fibrinolítico pode também ter sua contribuição ${ }^{5}$, já que são encontrados níveis elevados de fibrinopeptídeo $A$ e atividade anormal da trombina em pacientes com a síndrome ${ }^{15}$.

\section{Anormalidades pulmonares}

Além do embolismo pulmonar, outras manifestações pulmonares associadas à síndrome são: hipertensão pulmonar, hemangiomas cavernosos pleurais levando a hemotórax, obstrução linfática pulmonar e varicosidades de veias pulmonares.

\section{Linfedema e síncope}

A obstrução dos vasos linfáticos pode causar linfedema e é vista com frequência em pacientes com a síndrome de Klippel-Trenaunay. Entretanto, raramente essa é uma complicação debilitante ${ }^{8,14}$. O grande acúmulo de sangue na região venosa em membros inferiores pode levar a hipotensão ortostática e síncope, efeitos esses agravados na gravide $z^{37}$. 
Talans A, et al. Gestação em pacientes com síndrome de Klippel-Trenaunay.

Gestação e síndrome de Klippel-
Trenaunay

Foram descritos apenas 17 casos de gravidez em mulheres com síndrome de Klippel-Trenaunay na literatura (Quadro 1).

O primeiro caso foi descrito em 1989 por Verheijen et al. ${ }^{38}$.

São conhecidos os riscos que a síndrome de Klippel-Trenaunay provoca tanto para a gestante como para o feto ${ }^{13}$, incluindo-se: hemorragia secundária ao aumento dos hemangiomas e varizes, trombose superficial e profunda, coagulação intravascular disseminada, síndrome de Kasabach-Merritt (hemangioma e trombocitopenia) ${ }^{27}$ e anormalidades placentárias.

Por sua vez, a gestação pode causar exacerbação das manifestações prévias da síndrome de Klippel-Trenaunay, devido ao aumento de níveis hormonais, de peso e de volume sanguíneo ${ }^{38}$. Pode ocorrer, por exemplo, aumento dos hemangiomas e da hipertrofia de tecidos moles e ossos, causando sangramento e dor, respectivamente.

QUADRO 1: Resumo dos casos de gestação em pacientes com síndrome de Klippel-Trenaunay descritos na literatura (adaptado de Stein et al. ${ }^{37}$ )

\begin{tabular}{|c|c|c|c|}
\hline Autor & História materna & Evolução da gestação & Intervenções \\
\hline $\begin{array}{l}\text { Verheijen et al. }{ }^{38} \\
\text { (1989) }\end{array}$ & $\begin{array}{l}28 \text { anos G1 } \\
\text { - Múltiplos hemangiomas cutâneos } \\
\text { - TVP em MID }\end{array}$ & $\begin{array}{l}\text { - Angiomatose cervical e uterina } \\
\text { - Anemia } \\
\text { - Parto a termo } \\
\text { - Cesariana clássica } \\
\text { - Swan-ganz }\end{array}$ & $\begin{array}{l}\text { - Anticoagulação pós-parto } \\
\text { por } 6 \text { semanas } \\
\text { - Transfusão sanguínea }\end{array}$ \\
\hline $\begin{array}{l}\text { Jorgensen et al. }{ }^{21} \\
\text { (1994) }\end{array}$ & $\begin{array}{l}30 \text { anos G2P1 } \\
\text { - Hemangiomas em abdômen e períneo } \\
\text { - Filho com hemangiomas }\end{array}$ & $\begin{array}{l}\text { - USG do feto mostrando grande } \\
\text { aumento do pé esquerdo e cistos } \\
\text { - Aborto eletivo com } 23 \text { semanas }\end{array}$ & \\
\hline $\begin{array}{l}\text { Pollack et al. }{ }^{30} \\
\text { (1995) }\end{array}$ & $\begin{array}{l}25 \text { anos G1 } \\
\text { - Hipertrofia de perna direita } \\
\text { - Hemangiomas e varicosidades }\end{array}$ & $\begin{array}{l}\text { - Tromboflebite superficial com } 34 \\
\text { semanas } \\
\text { - Parto a termo (vaginal, com vácuo- } \\
\text { extrator) }\end{array}$ & $\begin{array}{l}\text { - Meias compressivas } \\
\text { - Analgesia oral }\end{array}$ \\
\hline $\begin{array}{l}\text { Gaiser et al. }{ }^{14} \\
(1995)\end{array}$ & $\begin{array}{l}23 \text { anos G1 } \\
\text { - Hemangioma em MID, dor e hipotensão ortos- } \\
\text { tática } \\
\text { - Hemangioma em hemicorpo direito ao nível de } \\
\text { L3-4 na parede abdominal anterior e dorso } \\
\text { - Hemangiomas vulvares }\end{array}$ & $\begin{array}{l}\text { - Hipotensão à epidural } \\
\text { - Hipotonia uterina responsiva à } \\
\text { metilergometrina } \\
\text { - Hemorragia intra-operatória com ane- } \\
\text { mia pós-operatória } \\
\text { - CIVD de baixo grau } \\
\text { - Sangramento pós-operatório da incisão } \\
\text { - Cesariana } \\
\text { - Incisão cutânea paramediana, devido a } \\
\text { hemangiomas }\end{array}$ & $\begin{array}{l}\text { - Suporte para CIVD } \\
\text { - Exploração cirúrgica do } \\
\text { local de incisão para hemos- } \\
\text { tasia }\end{array}$ \\
\hline $\begin{array}{l}\text { Neubert et al. }{ }^{27} \\
\text { (1995) }\end{array}$ & $\begin{array}{l}22 \text { anos G1 } \\
\text { - Hemangiomas em parede abdominal direita, } \\
\text { coxa, vulva e parede vaginal lateral } \\
\text { - Hemartrose em MID }\end{array}$ & $\begin{array}{l}\text { - aumento dos hemangiomas } \\
\text { - hematoma na ferida cirúrgica } \\
\text { - Síndrome de Kasabach-Merritt pós- } \\
\text { operatória } \\
\text { - Parto a termo } \\
\text { - Cesariana } \\
\text { - Incisão cutânea paramediana, devido } \\
\text { aos hemangiomas }\end{array}$ & $\begin{array}{l}\text { - } 2 \text { laparotomias exploradas } \\
\text { e limpeza do hematoma da } \\
\text { ferida cirúrgica } \\
\text { - Transfusão sanguínea } \\
\text { - Heparina intravenosa }\end{array}$ \\
\hline Fait et al. ${ }^{13}(1996)$ & $\begin{array}{l}31 \text { anos G2P1 } \\
\text { G1 } \\
\text { - Hemangioma, varicosidades e hipertrofia em MIE } \\
\text { G2 } \\
\text {-idem }\end{array}$ & $\begin{array}{l}\text { G1 } \\
\text { - Aumento da hipertrofia e TVP em MIE } \\
\text { - Infarto placentário e congestão vascular } \\
\text { - indução por RCF } \\
\text { - Cesariana por falha de indução } \\
\text { G2 } \\
\text { - A termo } \\
\text { - Cesariana }\end{array}$ & - Aspirina e HBPM via oral \\
\hline $\begin{array}{l}\text { Richards et al. }{ }^{32} \\
\text { (1997) }\end{array}$ & $\begin{array}{l}31 \text { anos G1 } \\
\text { - Linfedema e hipertrofia de MID } \\
\text { - Deformidade em pé direito }\end{array}$ & $\begin{array}{l}\text { - Angiomatose uterina } \\
\text { - Interrupção voluntária da gestação com } \\
10 \text { semanas via D\&C }\end{array}$ & $-\mathrm{D} \& \mathrm{C}$ \\
\hline
\end{tabular}


QUADRO 1: Resumo dos casos de gestação em pacientes com síndrome de Klippel-Tremaunay descritos na literatura (apaptado de Stein et al. ${ }^{37}$ )

Continuação

\begin{tabular}{|c|c|c|c|}
\hline Autor & História materna & Evolução da gestação & Intervenções \\
\hline $\begin{array}{l}\text { Dobbs et al. }{ }^{11} \\
\text { (1999) }\end{array}$ & $\begin{array}{l}23 \text { anos G1 } \\
\text { - Hipertrofia em MID } \\
\text { - Hemangioma em parede abdominal direita e MID } \\
\text { - TVP em MID } \\
\text { - DMs }\end{array}$ & $\begin{array}{l}\text { - Aparecimento de veias anormais no } \\
\text { abdômen, pelve e fundo uterino } \\
\text { - Indução de parto por Diabetes } \\
\text { - Parto vaginal }\end{array}$ & $\begin{array}{l}\text { - Ressonância magnética de } \\
\text { abdômen e pelve }\end{array}$ \\
\hline $\begin{array}{l}\text { Watermeyer et } \\
\text { al. }{ }^{39}(2002)\end{array}$ & $\begin{array}{l}21 \text { anos G1 } \\
\text { - Hemangioma e hipertrofia em glúteo e MIE }\end{array}$ & $\begin{array}{l}\text { - Aumento dos hemangiomas } \\
\text { - Varicosidades em vulva, vagina e cérvix } \\
\text { - Hemorragia intra-operatória de } 1500 \mathrm{~mL} \\
\text { - Cesariana }\end{array}$ & $\begin{array}{l}\text { - Transfusão sanguínea } \\
\text { - Enoxaparina profilática } \\
\text { - Meias elásticas } \\
\text { compressivas }\end{array}$ \\
\hline $\begin{array}{l}\text { Hergesell et al. }{ }^{19} \\
(2003)\end{array}$ & $\begin{array}{l}32 \text { anos G1 } \\
\text { - Hemangioma facial bilateral } \\
\text { - Hipotrofia de MSE e MIE e TVP em MIE }\end{array}$ & $\begin{array}{l}\text { - edema em MMII } \\
\text { - embolia pós-operatória } \\
\text { - cesariana com } 36 \text { semanas }\end{array}$ & $\begin{array}{l}\text { - HBPM profilática } \\
\text { - Meias elásticas compres- } \\
\text { sivas }\end{array}$ \\
\hline $\begin{array}{l}\text { Rebarber et al. }{ }^{31} \\
(2004)\end{array}$ & $\begin{array}{l}21 \text { anos G1 } \\
\text { - Varicosidades em MMII } \\
\text { - Sem sinais de presença de sistema venoso } \\
\text { profundo }\end{array}$ & $\begin{array}{l}\text { - Múltiplas embolias pulmonares } \\
\text { - RCF com } 38 \text { semanas } \\
\text { - Endometrite, ITU e bacteremia pós- } \\
\text { operatória } \\
\text { - Indução de trabalho de parto, por RCF } \\
\text { - Parto vaginal }\end{array}$ & $\begin{array}{l}\text { - Heparina } \\
\text { - Anticoagulação por } 6 \\
\text { semanas pós-parto } \\
\text { - Ampicilina e Sulbactam } 3 \\
\text { vezes ao dia }\end{array}$ \\
\hline $\begin{array}{l}\text { Rebarber et al. }{ }^{31} \\
(2004)\end{array}$ & $\begin{array}{l}32 \text { anos G5P0A4 } \\
\text { - Malformação vascular em MID e mama direita } \\
\text { - Embolias em MMII e amputação de hálux direito } \\
\text { - Heterozigota para mutação em protrombina }\end{array}$ & $\begin{array}{l}\text { - Intensa dor em MMII, necessitando de } \\
\text { opióides } \\
\text { - Varicosidades vulvo-vaginais } 36 \text { sema- } \\
\text { nas, obstruindo canal de parto } \\
\text { - Cesariana com } 36 \text { semanas }\end{array}$ & $\begin{array}{l}\text { - HBPM da confirmação da } \\
\text { gestação até } 6 \text { semanas } \\
\text { pós-parto }\end{array}$ \\
\hline $\begin{array}{l}\text { Rebarber et al. }{ }^{31} \\
(2004)\end{array}$ & $\begin{array}{l}29 \text { anos G1 } \\
\text { - Malformação capilar em MIE e mama esquerda } \\
\text { e hipertrofia de MIE com múltiplos TVP } \\
\text { - Esplenectomia } \\
\text { - Hemorroidectomia } \\
\text { - Heterozigota para mutação em protrombina }\end{array}$ & $\begin{array}{l}\text { - Varicosidades vulvo-vaginais } \\
\text { - Trombose e hemangioma em ferida } \\
\text { cirúrgica } \\
\text { - Cesariana devido à varicosidades }\end{array}$ & - HBPM durante gestação \\
\hline Stein et al. ${ }^{37}(2006)$ & $\begin{array}{l}35 \text { anos G4P1 } \\
\text { - Varicosidades em MID } \\
\text { - Hemangioma em lábio direito } \\
\text { - Crioressecção de neoplasia cervical intraepitelial } \\
\text { grau III }\end{array}$ & $\begin{array}{l}\text { - Comprimento cervical diminuído } \\
\text { - Trabalho de parto pré-termo } \\
\text { - Parto vaginal com vácuo-extrator por } \\
\text { bradicardia fetal }\end{array}$ & - Heparina profilática \\
\hline Stein et al..$^{37}(2006)$ & $\begin{array}{l}22 \text { anos G1 } \\
\text { - Hemangiomas em lábio direito, MID e pé direito } \\
\text { - Hipertrofia de pé esquerdo e MIE } \\
\text { - Dor crônica em MIE } \\
\text { - Hipotireoidismo }\end{array}$ & $\begin{array}{l}\text { - Hematoma em joelho direito } \\
\text { - Edema e dor em MID } \\
\text { - Hematúria assintomática } \\
\text { - Aumento da pressão arterial } \\
\text { - Indução de trabalho de parto } \\
\text { - Cesariana devido à falha em indução }\end{array}$ & $\begin{array}{l}\text { - Ressonância Magnética } \\
\text { - Doppler de MMII } \\
\text { - Heparina ante e pós-parto }\end{array}$ \\
\hline Stein et al. ${ }^{37}(2006)$ & $\begin{array}{l}16 \text { anos G1 } \\
\text { - Sangramento digestivo crônico secundário à } \\
\text { malformação arteriovenosa em sigmóide e reto } \\
\text { - Malformações arteriovenosas no ligamento largo, } \\
\text { miométrio, intestino delgado e gordura periretal }\end{array}$ & $\begin{array}{l}\text { - TVP em MID } \\
\text { - Parto via vaginal } \\
\text { - Laceração do sulco vaginal, } \\
\text { necessitando } 2 \text { cirurgias adicionais } \\
\text { - Perda de } 1000 \mathrm{~mL} \text { de sangue }\end{array}$ & $\begin{array}{l}\text { - Ressonância Magnética } \\
\text { - USG pré-natal } \\
\text { - transfusão de } 8 \text { unidades de } \\
\text { hemoglobina pós-parto } \\
\text { - anticoagulação terapêutica }\end{array}$ \\
\hline Stein et al. ${ }^{37}(2006)$ & $\begin{array}{l}30 \text { anos G1 } \\
\text { - Hemangiomas em MID e lábio direito } \\
\text { - Hemangioma cervical } \\
\text { G2 } \\
\text { - idem }\end{array}$ & $\begin{array}{l}\text { G1 } \\
\text { - Sangramento de hemangioma cervical } \\
\text { - Oligodrâmnio } \\
\text { - Cesariana } \\
\text { G2 } \\
\text { - Sem complicações } \\
\text { - Cesariana }\end{array}$ & $\begin{array}{l}\text { - Sutura de laceração cervical } \\
\text { - Tocólise com Sulfato de } \\
\text { Magnésio }\end{array}$ \\
\hline
\end{tabular}


Talans A, et al. Gestação em pacientes com síndrome de Klippel-Trenaunay.

\section{Recomendações de acompanhamento}

Devido aos diversos eventos adversos já reportados (como hemorragia maciça, coagulação intravascular, trombose, dor, restrição ao crescimento fetal e prematuridade), recomenda-se uma abordagem multidisciplinar no acompanhamento dessas gestantes ${ }^{37}$.

\section{Aconsellhamento pré-concepção}

Deve ser realizada anamnese completa da paciente, com destaque para os eventos tromboembólicos, infecções, hematúria, anemia crônica, sangramentos, linfedema, úlcera de estase e dor. Atenção especial deve ser dada a história de complicações obstétricas prévias, pois pode ajudar a prever complicações futuras. No exame físico, é recomendado realizar exame especular, para avaliar as manifestações da síndrome e auxiliar no planejamento do parto ${ }^{38}$.

\section{Cuidados pré-natais}

Já existem relatos de diagnóstico da síndrome de Klippel-Trenaunay por ultrassonografia, ainda na fase uterina ${ }^{18,29,33}$, sendo o mais precoce com 17 semanas de gestação ${ }^{29,33}$. A avaliação ultrassonográfica e por imagem (ressonância magnética) é recomendada não apenas para determinar o canal de parto, mas também para determinar o tipo de anestesia (a presença de malformações vasculares pode determinar a formação de hematomas espinhais, lesão neurológica e diátese hemorrágica) ${ }^{37}$.

Pacientes com história de trombose venosa profunda ou tromboembolismo pulmonar devem ser avaliados para confirmar ou descartar trombofilia. Nesses casos, deve-se considerar anticoagulação e o uso de filtro de veia cava. Ao laboratório, devem ser solicitados hemograma e perfil de coagulação, pelo risco de anemia e coagulopatia causado pelos hemangiomas e varizes ${ }^{32}$.

O controle da dor pode ser de difícil manejo, já que a exacerbação da hipertrofia de ossos e tecidos moles pode causar dor intensa e perda de mobilidade, fazendo com que um rígido controle da dor seja necessário ${ }^{33}$.

\section{Cuidados intraparto e via de parto}

Em pacientes com a síndrome de KlippelTrenaunay, a via de parto (cesárea ou parto normal) não é determinada apenas pela história obstétrica ou pela apresentação fetal, mas também pela presença de hemangiomas ou varizes uterinas, cervicais, vaginais, vulvares ou abdominais. $\mathrm{Na}$ escolha pela cesárea, o local da incisão é importante.
Anormalidades vasculares superficiais e profundas no abdome podem requerer incisão mediana ou paramediana, ao invés da tradicional incisão de Pfannestiel.

Até mesmo em pacientes com pequenos hemangiomas ou varizes, um sangramento pequeno pode causar hemorragias e coagulação intravascular disseminada. Por isso, deve-se fazer a tipagem sanguínea e pedir reserva de hemocomponentes antes da operação ${ }^{32}$.

Assim como a incisão da cesárea, o local de anestesia também deve ser escolhido cuidadosamente ${ }^{37}$.

\section{Pós-natal}

Os cuidados pós-natais devem ser orientados pelo curso obstétrico e pela manifestação da síndrome de Klippel-Trenaunay. É sabido que pacientes com síndrome de Klippel-Trenaunay possuem risco aumentado para trombose venosa profunda e outros fenômenos tromboembólicos ${ }^{5,15,23}$. Esse risco pode ser agravado pela gestação. Não há estudos prospectivos sobre recomendações específicas para administração de anticoagulação em pacientes gestantes com síndrome de KlippelTrenaunay. Devem ser discutidos com a paciente os riscos e benefícios de anticoagulação, após o qual a anticoagulação terapêutica deve ser considerada para pacientes com histórico de eventos tromboembólicos, e a anticoagulação profilática para pacientes que permaneçam acamadas ou tenham algum grau de restrição à mobilidade e para aquelas com grandes varizes ou hemangiomas em membros inferiores. Além disso, anticoncepção oral deve ser evitada nas pacientes com Síndrome de Klippel-Trenaunay ${ }^{37}$.

\section{CONCLUSÃO}

Analisando-se os relatos de gestação em pacientes com síndrome de Klippel-Trenaunay percebe-se que a maioria dos casos evoluiu sem maiores complicações, embora sejam escassos os relatos de gravidez em portadoras dessa síndrome. Há, no entanto, relatos de casos cujas gestantes apresentaram sérias complicações na evolução obstétrica, como de hemorragia intraoperatória ${ }^{14}$, coagulação intravascular disseminada ${ }^{14}$ e embolias pulmonares ${ }^{19,31}$. Os fatores que explicam a ausência ou presença de complicações, bem com a ampla variedade dessas, não são esclarecidos. O tempo de evolução da síndrome de Klippel-Trenaunay e, principalmente, a severidade da doença, parecem associar-se à evolução.

Embora sejam frequentes as complicações 
maternas, com exacerbação das manifestações prévias da síndrome, habitualmente não são observadas complicações fetais decorrentes da síndrome ou manifestação fetal da síndrome de Klippel-Trenaunay, exceção feita ao relato de Jorgensen et al. ${ }^{21}$.

Apesar de haver relatos de recorrências familiares, o risco de transmissão da síndrome da mãe para seu filho é desconhecido ${ }^{26}$.

O reconhecimento e entendimento da síndrome de Klippel-Trenaunay e cuidado multidisciplinar pré-natal, intra e pós-parto parecem ser fundamentais para a boa evolução e o bom desfecho da gestação nessas pacientes.

Talans A, Ramalho AS, Bortolotto MRFL, Francisco RPV, Zugaib M. Pregnancy in KlippelTrenaunay syndrome: review of literature. Rev Medicina (São Paulo). 2010 abr.-jun.;89(2):93100.

\begin{abstract}
Klippel-Trenaunay syndrome is a rare congenital anomaly of unknown etiology, characterized by capillary and venous malformations, and hypertrophy of bone and soft tissue. Cases of pregnancy in women with Klippel-Trenaunay syndrome are rare and usually associated with adverse perinatal outcomes. Objective: To resume knowledge about pregnancy in the Klippel-Trenaunay syndrome as well as the syndrome so these patients be readily recognized and receive proper care to have a successful pregnancy outcome. Method: It was performed a review of the English literature, in the MEDLINE and Cochrane Library bases until October 2009. Results: Only 17 case reports of pregnancy in women with Klippel-Trenaunay syndrome have been found. The evolutions of pregnant were variable and involve adverse events, with worsening of previous manifestations, bleeding, disseminated intravascular coagulation, thromboembolic events and pain. Conclusion: Pregnancy increases the risk of adverse events. Knowledge of the syndrome and multidisciplinary prenatal, intra and postpartum care are essential to successful outcome of pregnancy.
\end{abstract}

KEY WORDS: Klippel-Trenaunay-Weber syndrome/etiology; Congenital abnormalities pregnancy; High-risk.

\title{
REFERÊNCIAS
}

1. Aelvoet GE, Jorens PG, Roelen LM. Genetic aspects of the Klippel-Trenaunay syndrome. $\mathrm{Br} \mathrm{J}$ Dermatol. 1992;126:603-7.

2. Al-Salman MMS. Klippel-Trenaunay syndrome: clinical features, complications, \& management. Surg Today. 1997;27:735-40.

3. Atiyeh BS, Musharrafieh RS. Klippel-Trenaunay-type syndrome: an eponym for various expressions of the same entity. J Med. 1995;26:253-60.

4. Bakerville PA, Ackroyd JS, Browse NL. The etiology of the Klippel-Trenaunay syndrome. Ann Surg. 1985;202:624-7.

5. Baskerville PA, Ackroyd JS, Thomas LM, Browse NL. The Klippel-Trenaunay syndrome: clinical, radiological and haemodynamic features and management. $\mathrm{Br} \mathrm{J}$ Surg. 1985;72:232-6.

6. Berry SA, Peterson C, Mize W, Bloom K, Zachary C, Blasco P, Hunter D. Klippel-Trenaunay syndrome. Am J Med Genet. 1998;79:319-26.

7. Bliznak J, Staple TW. Radiology of angiodysplasias of the limb. Radiology. 1974;110:35-44.

8. Caparo PH, Fisher J, et al. Klippel-Trenaunay syndrome. Keisei Geka. 2002;109:2052-62.
9. Ceballos-Quintal JM, Pinto-Escalante X, Castillo-Zapata U. A new case of Klippel-Trenaunay-Weber syndrome: evidence of autosomal dominant inheritance. Am J Med Genet. 1996;63:426-7.

10. Cohen MM Jr. Klippel-Trenaunay syndrome. Am J Med Genet. 2000;93:171-5.

11. Dobbs $P$, Caunt $A$, Alderson TJ. Epidural analgesia in an obstetric patient with Klippel-Trenaunay syndrome. Br J Anaesth. 1999;82:144-146.

12. Dogan R, Faruk Dogan $O$, Oç $M$, Akata $D$, Gümüs B, Balkanci F. A rare vascular malformation: KlippelTrenaunay syndrome. J Cardiovasc Surg (Torino). 2003;44:95-100.

13. Fait G, Daniel $Y$, Kuperfermine MJ, Gull I, Peyser MR, Lessing JB. Klippel-Trenaunay-Weber syndrome associated with fetal growth restriction. Hum Reprod. 1996;11:2544-5.

14. Gaiser RR, Cheek TG, Gutsche BB. Major conduction anesthesia in a patient with Klippel-Trenaunay syndrome. J Clin Anesth. 1995;7:316-9.

15. Gianlupi A, Harper RW, Dwyre DM, Marelich GP. Recurrent pulmonary embolism associated with KlippelTrenaunay syndrome. Chest. 1999;115:1199-201. 
Talans A, et al. Gestação em pacientes com síndrome de Klippel-Trenaunay.

16. Gloviczki $P$, Hollier LH, Trelander RL, Kaufman B, Bianco AJ, Stickler GB. Surgical implications of KlippelTrenaunay syndrome. Ann Surg. 1983;197:353-62.

17. Happle R. Lethal genes surviving by mosaicism: a possible explanation for sporadic birth defects involving the skin. J Am Acad Dermatol. 1997;16:899-906.

18. Hatjis CG, Philip AG, Anderson GG, Mann LI. The in-utero ultrasonographic appearance of KlippelTrenaunay-Weber syndrome. Am J Obstet Gynecol. 1981;139:972-4.

19. Hergesell K, Kroger K, Petruschkat S, Santosa F, Herborn C, Rudofsky G. Klippel-Trenaunay syndrome and pregnancy. Int Angiol. 2003;22:194-8.

20. Jacob AG, Driscoll DJ, Shaughnessy WJ, Stanson AW, Clay RP, Gloviczki P. Klippel-Trenaunay syndrome. Spectrum and management. Mayo Clin Proc. 1998;73:28-36.

21. Jorgensen RJ, Darby B, Patterson R, Trimmer KJ. Prenatal diagnosis of the Klippel-Trenaunay-Weber syndrome. Prenat Diagn. 1994;14:989-92.

22. Klippel $M$, Trenaunay $P$. Naevus variquex osteohypertrophique. Arch Gen Med. 1900;3:641-72.

23. Lawlor F, Charles-Holmes S. Uterine haemangioma in Klippel-Trenaunay-Weber syndrome. J R Soc Med. 1988;81:665-6.

24. Lorda-Sanchez L, Prieto L, Rodriquez-Pinilla E, Martinez-Frias ML. Increased parental age and number of pregnancies in Klippel-Trenaunay-Weber syndrome. Ann Hum Genet. 1998;6:235-9.

25. McGrory BJ, Amadio PC. Klippel-Trenaunay syndrome: orthopaedic consideration. Orthop Rev. 1993;22:4150 .

26. Meine JG, Schwartz RA, Janniger CK. KlippelTrenaunay-Weber syndrome. Cutis. 1997;60:127-32.

27. Neubert AG, Golden MA, Rose NC. Kasabach-Merritt coagulopathy complication Klippel-TrenaunayWeber syndrome in pregnancy. Obstet Gynecol. 1995;85:831-3.

28. Nicolaides NA, Belcaro G, Goldberg D, et al. Prevention of thromboembolism: European Consensus Statement. In: Bergqvist D, Comerota AJ, Nicolaides NA, Scurr $\mathrm{JH}$, editors. Prevention of venous thromboembolism. London: Med-Orion Publishing Co.;199. p.445-56.

29. Paladini D, Lamberti A, Teodoro A, Liguori M,
D'Armiento M, Capuano $\mathrm{P}$, et al. Prenatal diagnosis and hemodynamic evaluation of Klippel-TrenaunayWeber syndrome. Ultrasound Obstet Gynecol. 1998;12:215-7.

30. Pollack RN, Quance DR, Shatz RM. Pregnancy complicated by the Klippel-Trenaunay syndrome. J Reprod Med. 1995;40:240-2.

31. Rebarber A, Roman AS, Roshan D, Roshan D, Blei F. Obstetric management of Klippel-Trenaunay syndrome. Obstet Gynecol. 2004;104:1205-8.

32. Richards DS, Cruz AC. Sonographic demonstration of widespread uterine angiomatosis in a pregnant patient with Klippel-Trenaunay-Weber syndrome. J Ultrasound Med. 1997;16:631-3.

33. Roberts RV, Dickinson JE, Hugo PJ, Barker A. Prenatal sonographic appearance of Klippel-Trenaunay-Weber syndrome. Prenat Diagn. 1999;19:369-71.

34. Servelle M. Klippel and Trenaunay's syndrome. Ann Surg. 1985;201:365-73.

35. Sethi S, Shubha. Klippel-Trenaunay syndrome. Indian J Pediatr. 2001;68:787-9.

36. Spitz SM. Klippel-Trenaunay syndrome: clinical features, complications, and management in children. Br J Surg. 1995;82:757-61.

37. Stein SR, Perlow JH, Sawai SK. Klippel-Trenaunay syndrome in pregnancy. Obstet Gynecol Surv. 2006;3:194-206.

38. Verheijen RHM, Van Rijen-de, Rooij HJM, van Zundert AAJ, et al. Pregnancy in a patient with KlippelTrenaunay-Weber syndrome: a case report. Eur $J$ Obstet Gynecol. 1989;33:89-94.

39. Watermeyer SR, Davies N, Goodwin RI. The Klippel-Trenaunay syndrome in pregnancy. BJOG. 2002;109:1301.

40. Whelan AJ, Watson MS, Porter FD, Steiner RD. Klippel-Trenaunay-Weber syndrome associated with a 5:11 balanced translocation. Am J Med Genet. 1995;59:492-4.

41. You CK, Rees J, Gillis DA, Steeves J. KlippelTrenaunay syndrome. Can J Surg. 1983;26:399-403.

Artigo recebido em: 23/04/2010

Artigo aceito em: 30/05/2010 